CERN-TH-2003/243

hep-th/0311244

\title{
Comments on Noncommutative Field Theories ${ }^{1}$
}

\author{
Luis Álvarez-Gaumé and Miguel A. Vázquez-Mozo² \\ Theory Division CERN \\ CH-1211 Geneva 23 \\ Switzerland \\ Luis.Alvarez-Gaume@cern.ch, Miguel.Vazquez-Mozo@cern.ch
}

\begin{abstract}
We discuss some aspects of noncommutative quantum field theories obtained from the Seiberg-Witten limit of string theories in the presence of an external $B$-field. General properties of these theories are studied as well as the phenomenological potential of noncommutative QED.
\end{abstract}

\footnotetext{
${ }^{1}$ Lectures presented at the 9th Adriatic Meeting in Dubrovnik (Croatia), September 2003 (L.A.-G.) and, in a shortened form, at String Phenomenology 2003 in Durham (U.K.) July 2003 (M.A.V.-M.).

${ }^{2}$ Address after January 1st, 2004: Física Teórica, Universidad de Salamanca, Plaza de la Merced s/n, E-37008 Salamanca, Spain.
} 


\section{Introduction}

Since its formulation by Alain Connes, noncommutative geometry (NCG) has become a very active and interesting branch of Mathematics [1]. In Physics, NCG has had an early impact in a number of subjects including condensed matter physics [2] and high energy physics [3]. In String Theory, the use of NCG was pioneered by its application by Witten to string field theory [4]. More recently, compactifications of string and Mtheory on noncommutative tori were studied in [5]. Although quantum field theories in noncommutative spaces had been the subject of attention [6], a renewed interest in the subject came after the realization by Seiberg and Witten [7] that a certain class of field theories on noncommutative Minkowski space can be obtained as particular low-energy limits in the presence of a constant NS-NS $B$-field.

Unlike the standard low-energy limit of string theory, the Seiberg-Witten limit leads to a nonlocal effective theory, where the interaction vertices are constructed in terms of the nonlocal Moyal product (see [8] for comprehensive reviews). In physical terms, this nonlocality is due to the extended nature of the low energy excitations, which in fact are rigid rodes whose size depends on the momentum of the state [9]. It is therefore interesting, from the field theoretic point of view, to understand how our ordinary view of field theory changes by the introduction of this particular type of nonlocality. Many standard notions and results require revision, like renormalizability, unitarity, discrete and space-time symmetries, etc. Nonetheless, since these theories are obtained from String Theory, one would expect them to be better behaved than other kinds of nonlocal theories.

One of the more remarkable results in the subject was obtained by Minwalla, van Raamsdonk and Seiberg [10]. These authors realized that quantum theories on noncommutative spaces are afflicted from an endemic mixing of ultraviolet (UV) and infrared (IR) divergences. Even in massive theories the existence of UV divergences induce IR problems, and this leads to a breakdown of the Wilsonian approach to field theory. Contrary to some expectations [11], noncommutativity does not provide a full regularization of UV divergences, but only of a subsector of the Feynman graphs. Hence the issue of renormalizability of NCQFT become rather subtle [12].

In ordinary Quantum Field Theory there are a number of properties that can be derived from general principles collectively called Wightman axioms [13]. Among them we can cite the CPT theorem, the connection between spin-statistics and the cluster decomposition. The extension of some of these properties to NCQFTs is not straightforward $[14,15,16]$ and therefore it would be interesting to study whether this kind of nonlocal field theories admit an axiomatic formulation in order to gain a better insight about the extension to NCQFTs of properties like the CPT and spin-statistics theorems [16].

In this lecture we would like to make a number of remarks on noncommutative field theories, in particular those obtained from String Theory through the Seiberg-Witten limit. We will pay special attention to the analysis of the phenomenological viability of this kind of field theories. For that we will focus on noncommutative QED (NCQED). The Standard Model contains Maxwell's theory at low energies and thus the "usual" photon should be recovered in any noncommutative generalization of QED, independently of how the Standard Model is embedded into its noncommutative extension. Among the properties of the QED photon, we will look at its masslessness, and the fact that 
the speed of light is constant, i.e. independent of the magnitude and direction of the photon momentum [17]. As we will see, it is remarkably difficult to obtain that ordinary electromagnetism is embedded as the low-energy limit of a noncommutative $U(1)$-theory. In particular, due to UV/IR mixing, it is rather common to obtain that one of the photon polarizations remains massless while the other becomes either massive or tachyonic. In ordinary gauge theories vector bosons get masses through the Higgs mechanism. Here the nonlocality of the interaction terms may lead to a massive photon polarization.

In order to give sense to NCQED we define it in terms of a softly broken $\mathcal{N}=4$ noncommutative $\mathrm{U}(1)$ gauge theory. This provides a construction that makes sense in the UV and IR, and where we can have control on the UV/IR mixing. Here we find that unless some conditions are satisfied by the soft breaking terms, one of the components of the photon becomes tachyonic. Even when this disaster is avoided, one generically gets a completely unacceptable value for the photon mass, unless one is willing to engage in massive fine-tuning. We will follow the presentation in our paper [16], where a more complete list of references is provided.

Before we proceed we would like to clearly state our point of view. As mentioned above, we will focus here on the type of noncommutative theories that are obtained from string theory via the Seiberg-Witten limit. There are of course other approaches to the problem, and we would like to briefly make a comparison. If one follows the quantization procedure proposed in Refs. [18, 19] the results should be the same, because both approaches agree in the case of space-space noncommutativity. Regarding the approach of Ref. [20], they extend the Seiberg-Witten map to arbitrary groups, and their actions are obtained order by order in an expansion in powers of $\theta$. Hence if we truncate at a given order, we find the standard commutative Lagrangian, and a collection of corrections corresponding the higher dimension operators. This theory is technically nonrenormalizable and one should not find UV/IR mixing, which occur only after one has summed to all order in $\theta$, in which case we would expect to obtain the same results because the Feynman rules are the same. Other approaches has been studied in [21].

We follow here the "orthodox" string approach, namely we use the Feynman rules that follow from String Theory after we take the Seiberg-Witten limit, in particular we restrict our considerations always to space-space noncommutativity. Since the vertices and Feynman integrands are only modified by sine and cosine functions, the naive degree of divergence of the theory will not change, and one should expect some sort of renormalizability to hold once the UV/IR problems are tamed. It is possible to extend the Seiberg-Witten limit to have time-space noncommutativity, but this does not lead to a field theory but a theory of noncommutative open string [22].

In the next section we give a short overview of some well-known facts about NCQFTs, in particular the UV/IR mixing characteristic of these theories. In Section 3 an extension of axiomatic formulation to NCQFTs is briefly discussed as well as the validity of the CPT theorem in this type of theories. Section 4 reviews the IR problems of NCQED and in Section 5 we study the construction of such a theory from its softly broken $\mathcal{N}=$ 4 supersymmetric extension and the possibility of eliminating tachyonic states in the spectrum. This section concludes with a discussion of the phenomenological prospects of NCQED. 


\section{Seiberg-Witten limit, dipoles and UV/IR mixing}

In [7] it was shown how noncommutative field theories are obtained as a particular lowenergy limit of open string theory on D-brane backgrounds in the presence of constant NS-NS $B$-field. In this case, the endpoints of the open strings behave as electric charges in the presence of an external magnetic field $B_{\mu \nu}$ resulting in a polarization of the open strings. Labeling by $i=1, \ldots, p$ the D-brane directions and assuming $B_{0 i}=0$, the difference between the zero modes of the string endpoints is given by [9]

$$
\Delta X^{i}=X^{i}(\tau, 0)-X^{i}(\tau, \pi)=\left(2 \pi \alpha^{\prime}\right)^{2} g^{i j} B_{j k} p^{k},
$$

where $g_{\mu \nu}$ is the closed string or $\sigma$-model metric and $p^{\mu}$ is the momentum of the string. In the ordinary low-energy limit, where $\alpha^{\prime} \rightarrow 0$ while $g_{\mu \nu}$ and $B_{\mu \nu}$ remain fixed, the distance $|\Delta X|$ goes to zero and the effective dynamics is described by a theory of particles, i.e. by a commutative quantum field theory.

There are, however, other possibilities of decoupling the massive modes without collapsing at the same time the length of the open strings. Seiberg and Witten proposed to consider a low-energy limit $\alpha^{\prime} \rightarrow 0$ where both $B_{i j}$ and the open string metric

$$
G_{i j}=-\left(2 \pi \alpha^{\prime}\right)^{2}\left(B g^{-1} B\right)_{i j}
$$

are kept fixed. Introducing the notation $\theta^{i j}=\left(B^{-1}\right)^{i j}$, the separation between the string endpoints can be expressed as:

$$
\Delta X^{i}=\theta^{i j} G_{j k} p^{k}
$$

fixed in the low-energy limit. The resulting low-energy effective theory is a noncommutative field theory with noncommutative parameter $\theta^{i j}$. In physical terms the SeibergWitten limit corresponds to making the string tension go to infinity, while and balancing it with the Lorentz force on the string-ends caused by the external magnetic field. This limit makes the string rigid and with a finite length that depends on its momentum.

The previous analysis was confined to situations in which the $B_{0 i}$ components are set to zero. The result is a noncommutative field theory with only space-space noncommutativity. From a purely field-theoretical point of view it is possible to consider also noncommutative theories where the time coordinate does not commute with the spatial ones, i.e. $\theta^{i 0} \neq 0$. In this case, however, non-locality is accompanied by a breakdown of unitarity reflected in the fact that the optical theorem is not satisfied [23, 24]. In addition there is no well-defined Hamiltonian formalism (see, however, the alternative approaches of $[18,19])$. From the string theory point of view, taking the Seiberg-Witten limit with $B_{0 i} \neq 0$ results in a lack of decoupling of closed string modes. In the resulting low energy noncommutative field theory the violation of the optical theorem can be formally solved by including the undecoupled string modes that, however, have negative norm [24]. In the following we will restrict our attention to the case of space-space noncommutativity, $\theta^{0 i}=0$.

In the Seiberg-Witten limit we obtain therefore field theories on a quantum plane, the coordinates $x^{\mu}$ do not commute but rather satisfy:

$$
\left[x^{\mu}, x^{\nu}\right]=i \theta^{\mu \nu}
$$


with

$$
\theta^{\mu \nu}=\left(\begin{array}{cccc}
0 & 0 & 0 & 0 \\
0 & 0 & \theta & 0 \\
0 & -\theta & 0 & 0 \\
0 & 0 & 0 & 0
\end{array}\right)
$$

The action for these field theories looks the same as for commutative theories except that functions are multiplied in terms of the Moyal product:

$$
f(x) \star g(x)=f(x) e^{\frac{i}{2} \theta^{\mu \nu} \overleftarrow{\partial}_{\mu} \vec{\partial}_{\nu}} g(x)
$$

Using the Fourier transform of (2.6) we can write down the Feynman rules for a scalar field theory containing a $\varphi_{\star}^{n}$-vertex. The result is:

$$
\int d^{d} x \varphi(x)_{\star}^{n}=\int \frac{d^{d} k_{1}}{(2 \pi)^{d}} \ldots \frac{d^{d} k_{n}}{(2 \pi)^{d}}(2 \pi)^{d} \delta\left(\sum_{j=1}^{n} k_{j}\right) \tilde{\varphi}\left(k_{1}\right) \ldots \tilde{\varphi}\left(k_{n}\right) e^{-\frac{i}{2} \sum_{i<j} k_{i} \cdot \tilde{k}_{j}}
$$

with $\tilde{k}^{\mu} \equiv \theta^{\mu \nu} k_{\nu}$ and $\tilde{\varphi}(k)$ the Fourier transform of $\varphi(x)$.

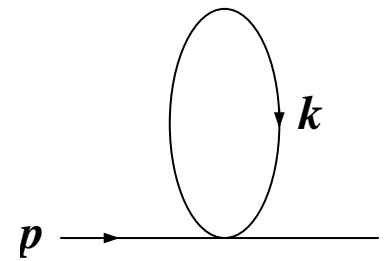

(a)

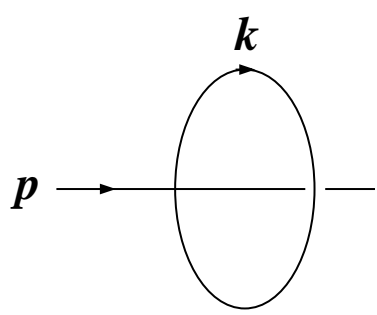

(b)

Figure 1: Planar (a) and nonplanar (b) contributions to the mass renormalization in $\lambda \varphi_{\star}^{4}$.

The phases in (2.7) are at the origin of the UV/IR mixing [10]. Because of these phases depend on the incoming momenta in the vertex, planar and nonplanar Feynman diagrams will have different contributions. As a matter of example one can consider the mass renormalization in $\lambda \varphi_{\star}^{4}$-theory. Whereas in the commutative theory there is a single diagram contributing to one loop, the new Feynman rules produce two contributions (see Fig. 1). One of them is the quadratically divergent planar diagram which is identical to the commutative one except for a different combinatorial factor. Together with this there is the "non-planar" contribution (see [10] for details) which has the form:

$$
\Pi(p)_{\text {nonplanar }}=\frac{\lambda}{6} \int \frac{d^{4} k}{(2 \pi)^{4}} \frac{e^{i k \cdot \tilde{p}}}{k^{2}+m^{2}} .
$$

As long as the external momentum $p$, or rather $\tilde{p}$, is nonvanishing, the integral converges due to the rapidly oscillating phase at large loop momentum. Exponentiating the denominator in (2.8) using a Schwinger parameter and introducing a Schwinger cutoff $\Lambda$ we find that the nonplanar diagram has an effective momentum-dependent cut-off given by

$$
\frac{1}{\Lambda_{\mathrm{eff}}^{2}}=\frac{1}{\Lambda^{2}}+\tilde{p}^{2},
$$


This clearly shows how UV divergences of (2.8) are transformed into IR ones. After the UV cutoff $\Lambda$ is sent to infinity, the quadratic divergence will reappear in the IR limit $\tilde{p} \rightarrow 0$. At fixed cutoff, on the other hand, the UV/IR mixing reflects in that the two limits $\Lambda \rightarrow \infty$ and $\theta \rightarrow 0$ do not commute. It is thus clear that in general we will have problems defining low-energy Wilsonian effective actions since UV and IR scales do not decouple.

The phenomenon of UV/IR mixing has some resemblance with Quantum Gravity or String Theory, and is probably one of the reasons why NCQFTs have received so much attention. When we consider General Relativity, an object with a given energy $E$ has two lengths associated with it: one is the Compton wavelength as in ordinary field theory $\hbar / E$. At the same time it also has its Schwarzschild radius $G_{N} E$. Obviously as the energy grows there is a point where the Schwarzschild radius becomes bigger than the Compton wavelength, and certainly at this point our standard notions of quantum field theory no longer apply.

If we consider the origin of the UV/IR mixing, the analogy is very appealing. In the loops of the NCQFTs we will have particles of very high energy and thus very short Compton wavelength. Since the fundamental objects in the theory are dipoles, the states running in loops have also an associated length of order $\theta p$, growing with the energy. If we define the theory with a sharp momentum space cutoff $\Lambda$, the longest dipole has size $\Lambda \theta$. This rod-like structure of the noncommutative quanta breaks Lorentz invariance, as it is already clear from the commutation relations (2.4) with the noncommutativity parameter given by (2.5). However if we consider momenta $0 \leq p \lesssim 1 /(\Lambda \theta)$ Lorentz invariance and the commutative theory should be recovered, since these momenta correspond to length scales bigger than the size of the largest dipole and therefore one cannot probe the "dipolar" structure of the excitations of the noncommutative theory.

\section{Residual symmetries, general properties and dis- crete symmetries}

Looking at the commutation relations (2.4) we see that NCQFTs are invariant under translations and therefore states can still be labeled by their four-momentum eigenvalues. On the other hand ${ }^{3}$, given the form of $\theta$ in Eq. (2.5), the Lorentz group is broken from $\mathrm{O}(1,3)$ to $\mathrm{O}(1,1) \times \mathrm{SO}(2)$.

For generic theories with these reduced symmetry one should not expect a connection between spin and statistics, and it should not be hard to construct theories with exotic statistics. It is thus an interesting question whether basic theorems like CPT which hold in local relativistic field theories will continue to hold in this context. Since we consider these theories as obtained from the Seiberg-Witten limit, it is reasonable to ask this question within String Theory. The CPT theorem in string theory has been investigated by several groups [25]. If the parent string theory satisfies the CPT theorem in perturbation theory, and since the constant background $B$-field is CPT-even, it is reasonable to expect that

\footnotetext{
${ }^{3}$ In the general case, $\theta^{\mu \nu}$ is determined by two vectors, the electric and magnetic components $\theta^{0 i}$ and $\epsilon^{i j k} \theta^{j k}$. If they are not collinear, the Lorentz group is completely broken.
} 
the noncommutative quantum field theory obtained in the Seiberg-Witten limit should also preserve CPT.

At the level of the noncommutative field theory it is also expected to have CPTinvariance for theories with $\theta^{0 i}=0$. As mentioned above, these theories preserve perturbative unitarity. In ordinary quantum field theory there is an intimate connection between unitarity and CPT-invariance. Indeed, if the condition of asymptotic completeness holds, it can be shown $[26,27]$ that the $S$-matrix can be written in terms of the CPT operator of the complete theory $\Theta$ and the corresponding one for the asymptotic theory $\Theta_{0}$ :

$$
S=\Theta^{-1} \Theta_{0}
$$

The unitarity of the $S$-matrix follows then from the antiunitarity of $\Theta$ and $\Theta_{0}$. A theory with CPT invariance is therefore likely to be unitary.

There are several proofs of the CPT theorem for ordinary QFTs. We find however that the deeper and more elegant one is due to Jost $[28,13]$. Few ingredients are required. One only needs the theory to satisfy the Wightman axioms, essentially Poincare invariance, uniqueness of the vacuum, positivity of the energy and microscopic causality. With these conditions it is shown that the Wightman functions ${ }^{4}$ admit an analytic continuation that is invariant under the complexified Lorentz group. The standard Lorentz group has four sheets, one connected to the identity, and the other three obtained by applying to it $\mathrm{P}, \mathrm{T}$ and PT. However, the complexified Lorentz group contains only two sheets, and the one obtained by applying the full space-time inversion (PT) is connected with the identity. Expressing invariance under this transformation essentially amounts to the proof of the CPT theorem.

In spite of the reduced space-time symmetry, this proof can be extended to NCQFTs, at least those with space-space noncommutativity ${ }^{5}$. The key ingredient lies in the fact that for NCQFT microcausality is defined only with respect to the $\mathrm{O}(1,1)$ factor of the space-time symmetry group. Given the fact that this group has a structure very similar to the full $\mathrm{O}(1,3)$ Lorentz group, Jost's proof is carried out to the noncommutative case without any problem (see [16] for the details).

Hence, although in general one should expect serious problems with nonlocal theories, for the type of nonlocality produced by the Seiberg-Witten limit we nevertheless recover the standard form of the CPT theorem. The spin-statistics connection is more subtle. If the type of representations of the reduced Lorentz group in NCQFTs are obtained as reductions from standard representations, it is likely that the same construction goes through. However, if we start with other representations, not inherited from higher dimensions, exotic statistics may easily occur.

\footnotetext{
${ }^{4}$ Wightman functions are vacuum expectation values of products of fields without time-ordering, namely

$$
W_{n}\left(x_{1}, \ldots, x_{n}\right)=\left\langle\Omega\left|\Phi\left(x_{1}\right) \ldots \Phi\left(x_{n}\right)\right| \Omega\right\rangle
$$

where $|\Omega\rangle$ is the true vacuum of the theory.

${ }^{5} \mathrm{~A}$ different proof that applies also the the time-space noncommutativity can be found in [14].
} 


\section{The infrared problems of noncommutative QED}

As we discussed in Section 2, much of the interesting physics of NCQFTs comes from $\mathrm{UV} / \mathrm{IR}$ mixing. This lack of decoupling of the different scales in the theory might pose a serious problem to phenomenology, since noncommutative effects can show up at low energies interfering with standard model predictions.

Since, apart from gravity, electromagnetism is the only long range interaction at low energies, it seems that QED would be the perfect test bench for the phenomenology of NCQFT. The noncommutative version of QED can be easily constructed by deforming ordinary QED with the introduction of Moyal products. Here we will consider the simplest case of pure NCQED with action

$$
S_{\mathrm{NCQED}}=\frac{1}{4 g^{2}} \int d^{4} x F_{\mu \nu} F^{\mu \nu}
$$

where $g$ is the coupling constant and

$$
F_{\mu \nu}=\partial_{\mu} A_{\nu}-\partial_{\nu} A_{\mu}-i\left(A_{\mu} \star A_{\nu}-A_{\nu} \star A_{\mu}\right)
$$

Because of the quadratic $\theta$-dependent term in the definition of $F_{\mu \nu}$, the noncommutative photon self-interacts unlike the case of ordinary QED. The corresponding Feynman rules are very similar to those of nonabelian Yang-Mills where the group structure constants are replaced by trigonometric functions of the incoming momenta (see Fig. 2).
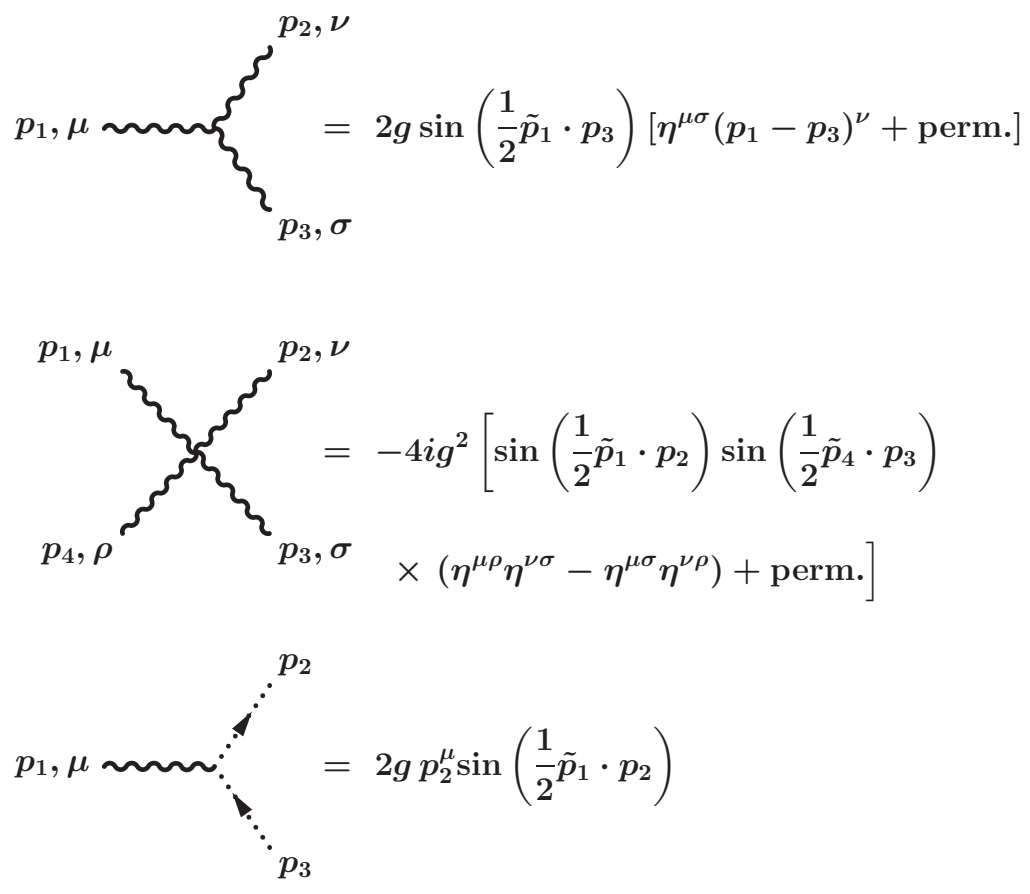

Figure 2: Interaction vertices for NCQED. The wavy line represents the photon and the line of points the Fadeev-Popov ghost.

At tree level, noncommutative corrections to ordinary QED can be made small and compatible with experimental bounds as long as the noncommutative energy scale $1 / \sqrt{\theta}$ 
is chosen large enough. This is due to the fact that noncommutativity only appears in the form of global $\theta$-dependent phases which have a smooth commutative limit. The situation is radically different once one-loop effects are taken into account, due to UV/IR mixing. The kind of problems encountered show up in the calculation of the one-loop corrected dispersion relation for the photon in NCQED which results to be [29]

$$
\omega(\vec{p})^{2}=\vec{p}^{2}-\frac{2 g^{2}}{\pi^{2}} \frac{1}{p \circ p}
$$

where $p \circ p \equiv \theta^{2}\left(p_{1}^{2}+p_{2}^{2}\right)$. The divergence at low transverse momentum in (4.3) is the result of a UV quadratic divergence that reappears in the IR as a result of UV/IR mixing. It seems therefore that the disastrous situation implied by the previous dispersion relation could be overcome by completing noncommutative QED with another theory softer in the UV.

This was attempted by embedding NCQED into $\mathcal{N}=1$ noncommutative supersymmetric QED and breaking supersymmetry softly at a scale $M$ by adding a mass term to the gaugino. The resulting dispersion relation, however, eliminates the IR divergence although it leaves behind a finite tachyonic mass for the photon [30]

$$
\omega(\vec{p})^{2} \approx \vec{p}^{2}-\frac{g^{2} M^{2}}{2 \pi^{2}}
$$

In spite of having removed the divergence, the situation has not improved at all, since the tachyonic mass of the photon only depends on the soft-breaking mass of the gaugino and not on the noncommutative parameter $\theta$. Therefore it cannot be made small by taking the noncommutative energy scale large. On the contrary if, for phenomenological reasons, we set $M \sim 1 \mathrm{TeV}$ we find a tachyonic photon with a mass much outside the current experimental bounds.

As we already mentioned, the source of all the trouble with NCQED is the mixing of UV and IR scales, which induces IR singularities in nonplanar amplitudes. One possible way to tame this problem is by defining the noncommutative theory with an sharp UV cutoff $\Lambda>1 / \sqrt{\theta}$. In this case, the noncommutative scale gets, in a sense, "corrected" due to one loop effects and noncommutative effects start being relevant already at scales of order $1 /(\theta \Lambda)$. Moreover, because of the regularization of the UV singularities provided by the cutoff, the commutative theory is recovered at scales $E \ll 1 /(\theta \Lambda)$, including its Lorentz invariance.

Therefore, it seems that this might provide a way to define NCQED at low energies avoiding the problems of the emergence of tachyons. Unfortunately [16], there are additional difficulties associated with this regularization scheme. In particular, apart from the lattice, a sharp cutoff $\Lambda$ of the type required (either a cutoff in momenta or a Schwinger cutoff) leads to violations of gauge invariance. This can only be avoided by considering "mixed" cutoffs which combines a sharp cutoff with dimensional regularization. In the case of NCQED this scheme works fine for the one-loop polarization tensor where gauge invariance is preserved and the result for ordinary QED recovered at low momentum [16]. Nevertheless, its extension to other amplitudes or higher loops is more problematic. 


\section{Some phenomenological considerations on NCQED}

A second alternative, that we will pursue here, is to ameliorate the IR problems of NCQED by looking for high energy completion of the theory which would be free of UV divergences. In particular, let us consider $\mathcal{N}=4 \mathrm{U}(1)$ noncommutative super-Yang-Mills, which is believed to be finite [31] as its commutative counterpart. In this case, instead of a single $\mathrm{U}(1)$ gauge vector field we have one $\mathcal{N}=1$ vector multiplet together with three scalar multiplets in the adjoint representation. NCQED can be then recovered at low energies by breaking supersymmetry softly by adding masses $M_{f}$ to the gauginos and $M_{s}$ to the scalars [32]. This provides a construction that makes sense in the UV and IR, and where we can have control on the UV/IR mixing.

With this setup, we can proceed to compute the one-loop polarization tensor for the photon $\Pi_{\mu \nu}(p)$. We will work in Euclidean signature and rotate back to Minkowski at the end of the calculation. On symmetry grounds it has the form

$$
\Pi_{\mu \nu}(p)=\Pi_{1}(p)\left(p^{2} \delta_{\mu \nu}-p_{\mu} p_{\nu}\right)+\Pi_{2}(p) \frac{\tilde{p}_{\mu} \tilde{p}_{\nu}}{\tilde{p}^{2}}
$$

where $\tilde{p}^{\mu}=\theta^{\mu \nu} p_{\nu}$. It is important to notice that, due to the antisymmetry of $\theta^{\mu \nu}$, the extra piece on the right-hand side in Eq. (5.1) is transverse and the Ward identity $p^{\mu} \Pi_{\mu \nu}(p)=0$ is satisfied.

Now we can proceed to compute the functions $\Pi_{1}(p)$ and $\Pi_{2}(p)$ at one loop for the theory with soft-breaking mass terms. Using the background field method [33] and working in dimensional regularization in the $\overline{\mathrm{MS}}$ scheme the results are

$$
\begin{aligned}
\Pi_{1}(p) & =\frac{1}{4 \pi^{2}} \int_{0}^{1} d x\left\{\left[4-(1-2 x)^{2}\right]\left[\frac{1}{2} \log \left(\frac{\Delta_{v}}{4 \pi \mu^{2}}\right)+K_{0}\left(\sqrt{\Delta_{v}}|\tilde{p}|\right)\right]\right. \\
& -\left[1-(1-2 x)^{2}\right] \sum_{f}\left[\frac{1}{2} \log \left(\frac{\Delta_{f}}{4 \pi \mu^{2}}\right)+K_{0}\left(\sqrt{\Delta_{f}}|\tilde{p}|\right)\right] \\
& \left.-\frac{1}{2}(1-2 x)^{2} \sum_{s}\left[\frac{1}{2} \log \left(\frac{\Delta_{s}}{4 \pi \mu^{2}}\right)+K_{0}\left(\sqrt{\Delta_{s}}|\tilde{p}|\right)\right]\right\},
\end{aligned}
$$

and

$$
\begin{aligned}
\Pi_{2}(p) & =-\frac{1}{\pi^{2}} \int_{0}^{1} d x\left[\Delta_{v} K_{2}\left(\sqrt{\Delta_{v}}|\tilde{p}|\right)-\sum_{f} \Delta_{f} K_{2}\left(\sqrt{\Delta_{f}}|\tilde{p}|\right)\right. \\
& \left.+\frac{1}{2} \sum_{s} \Delta_{s} K_{2}\left(\sqrt{\Delta_{s}}|\tilde{p}|\right)\right] .
\end{aligned}
$$

Here $\mu$ is the dimensional regularization energy scale and the subindices $v, f$ and $s$ indicate respectively the contributions from the vector-ghost system, fermions and scalars. In addition we have defined

$$
\begin{aligned}
\Delta_{v} & =x(1-x) p^{2}, \\
\Delta_{f} & =M_{f}^{2}+x(1-x) p^{2}, \\
\Delta_{s} & =M_{s}^{2}+x(1-x) p^{2},
\end{aligned}
$$


with $M_{f}, M_{s}$ the soft-breaking masses.

With Eqs. (5.2) and (5.3) we can easily compute the dispersion relation by looking at the poles of the full propagator $G_{\mu \nu}(p)$, once the one loop 1PI parts are resumed:

$$
G_{\mu \nu}(p)=\frac{i g^{2}}{p^{2}}\left[\mathbf{1}+\frac{-g^{2}}{p^{2}} \boldsymbol{\Pi}(p)+\left(\frac{-g^{2}}{p^{2}}\right)^{2} \boldsymbol{\Pi}(p)^{2}+\ldots\right]_{\mu \nu}
$$

where $\mathbf{1}$ is the $4 \times 4$ identity matrix and $\Pi(p)$ is a matrix notation for the polarization tensor in Eq. (5.1). After a straightforward calculation we find

$$
\begin{aligned}
G_{\mu \nu}(p) & =\frac{i g^{2} p_{\mu} p_{\nu}}{p^{2}}+\frac{i g^{2}}{p^{2}\left[1+g^{2} \Pi_{1}(p)\right]}\left(\delta_{\mu \nu}-\frac{p_{\mu} p_{\nu}}{p^{2}}\right) \\
& +\left\{\frac{i g^{2}}{p^{2}\left[1+g^{2} \Pi_{1}(p)\right]+g^{2} \Pi_{2}(p)}-\frac{i g^{2}}{p^{2}\left[1+g^{2} \Pi_{1}(p)\right]}\right\} \frac{\tilde{p}_{\mu} \tilde{p}_{\nu}}{\tilde{p}^{2}} .
\end{aligned}
$$

Unlike the case of ordinary QED in Eq. (5.6) we have two sources of poles in the full photon propagator. On the one hand we find the usual solution

$$
p^{2}\left[1+g^{2} \Pi_{1}(p)\right]=0,
$$

which gives rise to the usual massless dispersion relation for the photon, $p^{2}=0$. Together with this we also find a second pole associated with photon polarizations along the vector $\tilde{p}^{\mu}$ :

$$
p^{2}\left[1+g^{2} \Pi_{1}(p)\right]+g^{2} \Pi_{2}(p)=0 .
$$

In order to extract the dispersion relation we perform the rotation back to Minkowski signature by replacing $p^{2} \rightarrow-p^{2}$ and $\tilde{p}^{2} \rightarrow p \circ p$. Using the low momentum expansion of Eqs. (5.2) and (5.3) we find the dispersion relation for the polarizations along $\tilde{p}^{\mu}$ for low momentum

$$
\omega(\vec{p})^{2} \approx \vec{p}^{2}-\frac{g^{2}}{2 \pi^{2}}\left(\sum_{f} M_{f}^{2}-\frac{1}{2} \sum_{s} M_{s}^{2}\right) .
$$

Unlike the case in which NCQED is completed in the UV by $\mathcal{N}=1 \mathrm{U}(1)$ noncommutative super-Yang-Mills [30], here we can avoid a tachyonic photon by appropriately tuning the soft breaking masses in Eq. (5.9), i.e. by demanding

$$
\sum_{f} M_{f}^{2}-\frac{1}{2} \sum_{s} M_{s}^{2} \leq 0 .
$$

Unfortunately, a tuning of this quantity to zero does not result in a massless photon polarization, as we would like to recover at low energies. When the inequality (5.10) is saturated the leading term in the expansion of $\Pi_{2}(p)$ around $\vec{p}=0$ is negative, and we find a dispersion relation with negative energy squared for low momentum photons. Therefore one is forced to a finite value of the quantity on the left hand side of Eq. (5.10), i.e. to a 
massive photon polarization. Using the current bounds for the photon mass [34], one has to engage in a massive fine tuning of the soft breaking masses

$$
\frac{1}{2} \sum_{s} M_{s}^{2}-\sum_{f} M_{f}^{2} \lesssim 10^{-32} \mathrm{eV}^{2}
$$

This result is not affected by the addition of matter in the fundamental representation of $\mathrm{U}(1)$. In the calculation of the one-loop polarization tensor fundamental fields in the loop only contribute to planar diagrams. Since the function $\Pi_{2}(p)$ in Eq. (5.1) is solely determined by non-planar diagrams the only effect of the fundamental fields is in modifying the running of the coupling constant through the function $\Pi_{1}(p)$.

Even if the problem of a tachyonic photon can be avoided by this un-natural fine tuning of the mass scales, the dispersion relation of photons with polarizations along $\tilde{p}^{\mu}$ will be different from the standard relation $\omega(\vec{p})=|\vec{p}|$ of photons with polarizations orthogonal to $\tilde{p}^{\mu}$. This implies that in the construction of NCQED we are studying here there is a phenomenon of birefringence associated with the fact that the dispersion relations (and therefore the speed of propagation) of photons with different polarizations are different (cf. [35]).

After our analysis we have to conclude that the phenomenological perspectives of NCQED look rather poor. In our attempt to eliminate the tachyonic polarization of the photon we have been lead to massive photon polarizations and birefringence, at the prize also of a huge fine tuning of the masses of the soft breaking masses.

To summarize, here we have studied the problem of making sense out of NCQED at low energies, as derived from string theory in the Seiberg-Witten limit. To ameliorate the hard IR problems that afflict this theory we have completed it in the UV by $\mathcal{N}=4$ noncommutative $\mathrm{U}(1)$ super-Yang-Mills, softly broken by mass terms for the gauginos and scalars. Our conclusions regarding the phenomenological viability of such a theory are, however, rather negative. We found that tachyons can be avoided only by allowing a massive polarization for the photon. This requires also a tremendous fine tuning of the soft-breaking masses. It seems, therefore, that any attempt to extract phenomenology from this theory should be postponed to find a formulation of the theory that can describe at least the rough features of the world we live in.

\section{Acknowledgments}

We thank the organizers of the 9th Adriatic Meeting and the String Phenomenology 2003 Conference for the opportunity of presenting this work. We are also thankful to J.L.F. Barbón, M. Chaichian, J.M. Gracia-Bondía, K.E. Kunze, D. Lüst, R. Stora and J. Wess for useful discussions. M.A.V.-M. acknowledges support from Spanish Science Ministry Grant FPA2002-02037. 


\section{References}

[1] A. Connes, Noncommutative Geometry, Academic Press 1994.

J. Madore, An Introduction to Noncommutative Differential Geometry and its Applications, Cambridge 1995.

G. Landi, An Introduction to Noncommutative Spaces and their Geometries, Springer 1997 [arXiv:hep-th/9701078]

J. M. Gracia-Bondía, J. C. Varilly and H. Figueroa, Elements of Noncommutative Geometry, Birkhäuser 2001.

[2] J. Bellisard, A. van Elst and H. Schulz-Baldes, The Noncommutative Geometry of the Quantum Hall Effect, [arXiv:cond-mat/9411052].

[3] A. Connes and J. Lott, Nucl. Phys. Proc. Suppl. 18B (1991) 29.

C. P. Martin, J. M. Gracia-Bondia and J. C. Varilly, Phys. Rept. 294 (1998) 363 [arXiv:hep-th/9605001].

[4] E. Witten, Nucl. Phys. B268 (1986) 253.

[5] A. Connes, M. R. Douglas and A. Schwarz, J. High Energy Phys. 02 (1998) 003 [arXiv:hep-th/9711162].

M. R. Douglas and C. M. Hull, J. High Energy Phys. 9802 (1998) 008 [arXiv:hepth/9711165].

[6] A. González-Arroyo and C. P. Korthals Altes, Phys. Lett. B131 (1983) 396.

T. Filk, Phys. Lett. B376 (1996) 53.

[7] N. Seiberg and E. Witten, J. High Energy Phys. 09 (1999) 032 [arXiv:hepth/9908142].

[8] M. R. Douglas and N. A. Nekrasov, Rev. Mod. Phys. 73 (2001) 977 [arXiv:hepth/0106048].

R. J. Szabo, Phys. Rept. 378 (2003) 207 [arXiv:hep-th/0109162].

[9] M. M. Sheikh-Jabbari, Phys. Lett. B455 (1999) 129 [arXiv:hep-th/9901080].

[10] S. Minwalla, M. van Raamsdonk and N. Seiberg, J. High Energy Phys. 02 (2000) 020 [arXiv:hep-th/9912072].

M. van Raamsdonk and N. Seiberg, J. High Energy Phys. 03 (2000) 035 [arXiv:hepth/0002186].

[11] H. S. Snyder, Phys. Rev. 71 (1947) 38.

[12] M. M. Sheikh-Jabbari, J. High Energy Phys. 06 (1999) 015 [arXiv:hep-th/9903107]. T. Krajewski and R. Wulkenhaar, Int. J. Mod. Phys. A15 (2000) 1011 [arXiv:hepth/9903187].

I. Chepelev and R. Roiban, J. High Energy Phys. 05 (2000) 037 [arXiv:hepth/9911098].

A. Armoni, Nucl. Phys. B593 (2001) 229 [arXiv:hep-th/0005208]. 
E. A. Nicholson, Perturbative Wilsonian formalism for noncommutative gauge theories in the matrix representation, PhD. Thesis, arXiv:hep-th/0305044.

[13] R. Jost, The General Theory of Quantized Fields, AMS 1965.

R. F. Streater and A. S. Wightman, PCT, Spin and Statistics and All That, Benjamin 1964.

R. Haag, Local Quantum Physics: Fields, Particles, Algebras, Springer 1992.

[14] M. Chaichian, K. Nishijima and A. Tureanu, Phys. Lett. B568 (2003) 146 [arXiv:hepth/0209008].

[15] M. M. Sheikh-Jabbari, Phys. Rev. Lett. 84 (2000) 5265 [arXiv:hep-th/0001167].

S. M. Carroll, J. A. Harvey, V. A. Kostelecky, C. D. Lane and T. Okamoto, Phys. Rev. Lett. 87 (2001) 141601 [arXiv:hep-th/0105082].

P. Aschieri, B. Jurčo, P. Schupp and J. Wess, Nucl. Phys. B651 (2003) 45 [arXiv:hepth/0205214].

[16] L. Álvarez-Gaumé and M. A. Vázquez-Mozo, Nucl. Phys. B668 (2003) 293 [arXiv:hep-th/0305093].

[17] S. M. Carroll, G. B. Field and R. Jackiw, Phys. Rev. D41 (1990) 1231.

S. R. Coleman and S. L. Glashow, Phys. Lett. B405 (1997) 249 [arXiv:hepph/9703240]; Phys. Rev. D59 (1999) 116008 [arXiv:hep-ph/9812418].

[18] S. Doplicher, K. Fredenhagen and J. E. Roberts, Commun. Math. Phys. 172 (1995) 187 [arXiv:hep-th/0303037].

D. Bahns, S. Doplicher, K. Fredenhagen and G. Piacitelli, Phys. Lett. B533 (2002) 178 [arXiv:hep-th/0201222].

D. Bahns, S. Doplicher, K. Fredenhagen and G. Piacitelli, Commun. Math. Phys. 237 (2003) 221 [arXiv:hep-th/0301100].

[19] Y. Liao and K. Sibold, Eur. Phys. J. C25 (2002) 469 [arXiv:hep-th/0205269]; ibid. C25 (2002) 479 [arXiv:hep-th/0206011].

[20] J. Madore, S. Schraml, P. Schupp and J. Wess, Eur. Phys. J. C16 (2000) 161 [arXiv:hep-th/0001203].

B. Jurčo, L. Möller, S. Schraml, P. Schupp and J. Wess, Eur. Phys. J. C21 (2001) 383 [arXiv:hep-th/0104153].

[21] A. Bichl, J. Grimstrup, H. Grosse, L. Popp, M. Schweda and R. Wulkenhaar, J. High Energy Phys. 0106 (2001) 013 [arXiv:hep-th/0104097].

A. A. Bichl, J. M. Grimstrup, H. Grosse, E. Kraus, L. Popp, M. Schweda and R. Wulkenhaar, Eur. Phys. J. C24 (2002) 165 [arXiv:hep-th/0108045].

H. Bozkaya, P. Fischer, H. Grosse, M. Pitschmann, V. Putz, M. Schweda and R. Wulkenhaar, Eur. Phys. J. C29 (2003) 133 [arXiv:hep-th/0209253].

[22] N. Seiberg, L. Susskind and N. Toumbas, J. High Energy Phys. 06 (2000) 021 [arXiv:hep-th/0005040].

R. Gopakumar, J. M. Maldacena, S. Minwalla and A. Strominger, J. High Energy 
Phys. 06 (2000) 036 [arXiv:hep-th/0005048].

J. L. F. Barbón and E. Rabinovici, Phys. Lett. B 486 (2000) 202 [arXiv:hepth/0005073].

R. G. Cai and N. Ohta, Prog. Theor. Phys. 104 (2000) 1073 [arXiv:hep-th/0007106].

[23] J. Gomis and T. Mehen, Nucl. Phys. B591 (2000) 265 [arXiv:hep-th/0005129].

O. Aharony, J. Gomis and T. Mehen, J. High Energy Phys. 09 (2000) 023 [arXiv:hepth/0006236].

[24] L. Álvarez-Gaumé, J. L. F. Barbón and R. Zwicky, J. High Energy Phys. 05 (2001) 057 [arXiv:hep-th/0103069].

[25] H. Sonoda, Nucl. Phys. B326 (1989) 135.

V. A. Kostelecky and R. Potting, Nucl. Phys. B359 (1991) 545.

A. Pasquinucci and K. Roland, Nucl. Phys. B473 (1996) 31 [arXiv:hep-th/9602026].

[26] C. N. Yang and D. Feldman, Phys. Rev. 79 (1950) 972.

[27] R. Haag, Phys. Rev. 112 (1958) 669.

D. Ruelle, Helv. Phys. Acta 35 (1962) 147.

[28] R. Jost, Helv. Phys. Acta 30 (1957) 409.

[29] F. Ruiz Ruiz, Phys. Lett. B502 (2001) 274 [arXiv:hep-th/0012171].

K. Landsteiner, E. López and M. H. G. Tytgat, J. High Energy Phys. 06 (2001) 055 [arXiv:hep-th/0104133].

[30] C. E. Carlson, C. D. Carone and R. F. Lebed, Phys. Lett. B549 (2002) 337 [arXiv:hep-ph/0209077].

[31] I. Jack and D. R. Jones, Phys. Lett. B514 (2001) 401 [arXiv:hep-th/0105221].

[32] J. G. Taylor, Phys. Lett. B121 (1983) 386.

J. J. van der Bij and Y. P. Yao, Phys. Lett. B125 (1983) 171.

[33] V. V. Khoze and G. Travaglini, J. High Energy Phys. 01 (2001) 026 [arXiv:hepth/0011218].

[34] K. Hagiwara et al. [Particle Data Group Collaboration], Phys. Rev. D66 (2002) 010001.

[35] Z. Guralnik, R. Jackiw, S. Y. Pi and A. P. Polychronakos, Phys. Lett. B517 (2001) 450 [arXiv:hep-th/0106044]. 